LMU-TPW 2000-06

MPI-PhT/2000-08

\title{
Structure of the Three-dimensional Quantum Euclidean Space
}

\author{
B.L. Cerchiai ${ }^{1,2}$, J. Madore ${ }^{2,3}$, S. Schraml ${ }^{1,2}$, J. Wess ${ }^{1,2}$ \\ ${ }^{1}$ Sektion Physik, Universität München \\ Theresienstr. 37, D-80333 München \\ ${ }^{2}$ Max-Planck-Institut für Physik \\ Föhringer Ring 6, D-80805 München \\ ${ }^{3}$ Laboratoire de Physique Théorique \\ Université de Paris-Sud, Bâtiment 211, F-91405 Orsay
}

\begin{abstract}
As an example of a noncommutative space we discuss the quantum 3dimensional Euclidean space $\mathbb{R}_{q}^{3}$ together with its symmetry structure in great detail. The algebraic structure and the representation theory are clarified and discrete spectra for the coordinates are found. The $q$-deformed Legendre functions play a special role. A completeness relation is derived for these functions.
\end{abstract}




\section{Introduction}

Noncommutative space-time structures are finding increasing interest in gauge theories. Special examples [1] have been discussed in the literature. Among them are

1) The canonical structure:

$$
\left[\hat{x}^{i}, \hat{x}^{j}\right]=i \Theta^{i j}, \quad \Theta^{i j} \in \mathbb{C}
$$

2) the Lie-algebra structure:

$$
\left[\hat{x}^{i}, \hat{x}^{j}\right]=i c_{k}^{i j} \hat{x}^{k}, \quad c_{k}^{i j} \in \mathbb{C}
$$

3) The quantum space structure:

$$
\left[\hat{x}^{i}, \hat{x}^{j}\right]=i c_{k l}^{i j} \hat{x}^{k} \hat{x}^{l}, \quad c_{k l}^{i j} \in \mathbb{C}
$$

We shall discuss a special example of the third case [2]. For a more general review of the quantum space structure see [3].

In all of the above cases we consider the associative algebra freely generated by the elements $\hat{x}^{i}$ modulo the respective relations. This algebra of formal power series forms the algebra $\mathcal{A}_{x}$.

$$
\mathcal{A}_{x}=\frac{\mathbb{C}\left[\left[\hat{x}^{1} \ldots \hat{x}^{N}\right]\right]}{R}
$$

For a physicist this means that he is free to use the relations to reorder the elements of an arbitary power series. For the quantum space algebra we wish to exclude pathological cases such as the trivial case where there is no relation at all or where the product of any two elements is zero modulo the relations. To exclude such cases we shall demand the Poincaré-Birkhoff-Witt property for the algebra. By this we mean that the dimension of the space of homogeneous polynomials is the same as in the commutative case. For the first and second examples this will be the case, for the third example we require the Yang-Baxter equation. To formulate it we write the relations in the form:

$$
\hat{x}^{i} \hat{x}^{j}=\hat{R}_{k l}^{i j} \hat{x}^{k} \hat{x}^{l}, \quad \hat{R}_{k l}^{i j} \in \mathbb{C}
$$

and define $N^{3} \times N^{3}$ matrices

$$
\begin{aligned}
& \hat{R}_{12 j_{1} j_{2} j_{3}} i_{1} i_{2} i_{3} \\
& \hat{R}_{23 j_{1} j_{2} j_{3}}^{i_{1} i_{2} i_{3}}=\hat{R}_{j_{1} j_{2}}^{i_{1} i_{2}} \delta_{j_{3}}^{i_{3}} \\
& \delta_{j_{1}}^{i_{1}} \hat{R}_{j_{2} j_{3}}^{i_{2} i_{3}}
\end{aligned}
$$

The Yang-Baxter equation is:

$$
\hat{R}_{12} \hat{R}_{23} \hat{R}_{12}=\hat{R}_{23} \hat{R}_{12} \hat{R}_{23}
$$

There are several known solutions of this equation. We are interested in such relations that allow a conjugation which makes the algebra a $*$-algebra. This is because we have to associate the observables like the coordinates with selfadjoint operators in a Hilbertspace. The $\hat{R}$-matrices for the quantum groups $S O_{q}(n)$ allow such conjugations.

The quantum space algebra is a comodule of a quantum group. We start from co-algebra relations

$$
\Delta\left(x^{i}\right)=T_{k}^{i} \otimes \hat{x}^{k}
$$

and compute

$$
\begin{aligned}
\Delta\left(x^{i}\right) \Delta\left(x^{j}\right) & =T_{k}^{i} T_{l}^{j} \otimes \hat{x}^{k} \hat{x}^{l} \\
& =T_{k}^{i} T_{l}^{j} \otimes \hat{R}_{m n}^{k l} \hat{x}^{m} \hat{x}^{n}
\end{aligned}
$$


If we demand $R T T$ relations

$$
T_{k}^{i} T_{l}^{j} \hat{R}_{m n}^{k l}=\hat{R}_{k l}^{i j} T_{m}^{k} T_{n}^{l}
$$

for the $T$-algebra, we find

$$
\Delta\left(x^{i}\right) \Delta\left(x^{j}\right)=\hat{R}_{k l}^{i j} \Delta\left(x^{k}\right) \Delta\left(x^{j}\right)
$$

There is always a solution to the $R T T$ relations given by

$$
T_{j}^{i}=\delta_{j}^{i}
$$

If this is the only solution then the bialgebra consists of the unit element only; not very interesting. If the $\hat{R}$-matrix leads to the quantum group $S O_{q}(n)$, we have a more interesting case. Instead of introducing the quantum group $S O_{q}(n)$ we shall deal with the corresponding $q$-Lie algebra $s o_{q}(n)$. The quantum space is then a module of this algebra.

In this paper we discuss the 3-dimensional case in great detail. The algebra is introduced in Chapter 2. It has a peculiar property, there is a homomorphism of the algebra $s o_{q}(3)$ into the algebra $\mathbb{R}_{q}^{3}$. This is discussed in Chapter 3 . The full algebra can then be generated by a central element, the radius $R$, and elements of the tensor product of an $s u_{q}(2)$ algebra and an $s u_{q}(1,1)$ algebra. The generators of the $s u_{q}(2)$ algebra are further restricted by relations that when the algebra is represented lead to a unique infinite-dimensional representation of $s u_{q}(2)$. We call this algebra the $t$-algebra. The $s u_{q}(1,1)$ algebra we call $K$-algebra. If we then demand that the $s o_{q}(3)$ algebra corresponds to orbital angular momentum the $K$ algebra is restricted in the same sense as the $t$-algebra. This is discussed in Chapter 4. This clarifies the algebraic structure of the $s o_{q}(3)$ module $\mathbb{R}_{q}^{3}$.

To discuss physics we need representations of the algebra. The observables should be represented by (essentially) self-adjoint linear operators in a Hilbert space. This way we can use the well developped formalism of quantum mechanics and its interpretation scheme. In Chapter 5 we discuss the representations of the algebra. We find that they are characterized by one real parameter $z_{0}$. In all these representations we obtain a discrete spectrum for the coordinate $X^{3}$, which along with $R$ and $T_{\text {orb }}^{3}$, the third component of the orbital angular momentum, form a complete commuting set of observables. The scale of the spacing of the eigenvalues of $X^{3}$ is determined by the constant $z_{0}$, the eigenvalues are exponentially spaced. This we call a $q$-lattice. We are not surprised that noncommuting variables lead to a discretization (latticization) of space 助.

In Chapter 6 we construct the transformation that leads to a basis where $\vec{T}_{o r b}^{2}$ is diagonal. The corresponding transformation function turn out to be the $q$-deformed associated Legendre functions. They are defined in Appendix D in terms of the big $q$-Jacobi polynomials. They satisfy a difference equation, a recursion equation and have orthogonaltity properties - in complete analogy to the usual associated Legendre functions. From the self-adjointness property of $X^{3}$ we derive a completeness relation as well, this is done in Appendix 国. Appendices A, B and $\mathrm{G}$ are devoted to the representation of the $s u_{q}(2)$ and $s u_{q}(1,1)$ algebras and their comultiplication.

\section{The Algebra of the Euclidean Quantum Space $\mathbb{R}_{q}^{3}$}

This algebra has been discussed in Ref [5], we use the same notation here: $\mathbb{R}_{q}^{3}$ :

$$
\begin{aligned}
X^{3} X^{+}-q^{2} X^{+} X^{3} & =0 \\
X^{3} X^{-}-q^{-2} X^{-} X^{3} & =0 \\
X^{-} X^{+}-X^{+} X^{-} & =\lambda X^{3} X^{3}, \quad \lambda=q-q^{-1}, \quad q \in \mathbb{R} .
\end{aligned}
$$


We shall assume $q>1$ in this paper. This non-commutative structure is our model for a non-commutative space. We can impose conjugation properties that are compatible with the relations (2.1) justifying the ' $\mathbb{R}^{\prime}$ in $\mathbb{R}_{q}^{3}$ :

$$
\overline{X^{+}}=-q X^{-}, \quad \overline{X^{3}}=X^{3} .
$$

The quantum space $\mathbb{R}_{q}^{3}$ has a co-module structure under the action of the quantum group $S O_{q}(3)$ [3] and a module structure under the corresponding $q$-Lie algebra. $s u_{q}(2)$ :

$$
\begin{aligned}
q^{-1} T^{+} T^{-}-q T^{-} T^{+} & =T^{3} \\
q^{2} T^{3} T^{+}-q^{-2} T^{+} T^{3} & =\left(q+q^{-1}\right) T^{+} \\
q^{2} T^{-} T^{3}-q^{-2} T^{3} T^{-} & =\left(q+q^{-1}\right) T^{-}
\end{aligned}
$$

The conjugation properties justifying the ' $u$ ' in $s u_{q}(2)$ are:

$$
\overline{T^{+}}=\frac{1}{q^{2}} T^{-}, \quad \overline{T^{3}}=T^{3} .
$$

The module structure that was found in Ref. [5] is:

$$
\begin{aligned}
T^{3} X^{3} & =X^{3} T^{3} \\
T^{3} X^{+} & =q^{-4} X^{+} T^{3}+q^{-1}\left(1+q^{-2}\right) X^{+} \\
T^{3} X^{-} & =q^{4} X^{-} T^{3}-q\left(1+q^{2}\right) X^{-} \\
& \\
T^{+} X^{3} & =X^{3} T^{+}+q^{-2} \sqrt{1+q^{2}} X^{+} \\
T^{+} X^{+} & =q^{-2} X^{+} T^{+} \\
T^{+} X^{-} & =q^{2} X^{-} T^{+}+q^{-1} \sqrt{1+q^{2}} X^{3} \\
& \\
T^{-} X^{3} & =X^{3} T^{-}+q \sqrt{1+q^{2}} X^{-} \\
T^{-} X^{+} & =q^{-2} X^{+} T^{-}+\sqrt{1+q^{2}} X^{3} \\
T^{-} X^{-} & =q^{2} X^{-} T^{-}
\end{aligned}
$$

In the limit $q=1$ we obtain from relations (2.1)-(2.7) the commutative $\mathbb{R}^{3}$ with the Lie algebra $s o(3)$ acting on it.

As a consequence of the above relations it follows that there is a central hermitean element, the $q$-deformed radius:

$$
\begin{aligned}
& R^{2}=X^{3} X^{3}-q X^{+} X^{-}-\frac{1}{q} X^{-} X^{+}=q^{2} \overline{X^{3}} X^{3}+\left(1+q^{-2}\right) \overline{X^{+}} X^{+}, \\
& \overline{R^{2}}=R^{2} .
\end{aligned}
$$

'Central' means that $R^{2}$ commutes with all the elements $X$ and $T$.

There is a well-known Casimir operator for the $s u_{q}(2)$ algebra:

$$
\vec{T}^{2}=\frac{q^{2}}{\lambda^{2}} \tau^{\frac{1}{2}}+\frac{1}{\lambda^{2}} \tau^{-\frac{1}{2}}+\tau^{-\frac{1}{2}} T^{+} T^{-}-\frac{1+q^{2}}{\lambda^{2}} .
$$

We have introduced the group-like element

$$
\tau=1-\lambda T^{3}
$$

and the elements $\tau^{\frac{1}{2}}$ and $\tau^{-\frac{1}{2}}$ as an extension of the algebra. We shall extend the algebra by the element $R=\left(R^{2}\right)^{\frac{1}{2}}$ and $R^{-1}=\left(R^{2}\right)^{-\frac{1}{2}}$ as well. 
The $\tau X$ and $\tau T$ commutation relations can be obtained from the $T^{3} X$ and $T^{3} T$ relations and vice versa. They are

$$
\begin{aligned}
\tau X^{3} & =X^{3} \tau \\
\tau X^{+} & =q^{-4} X^{+} \tau \\
\tau X^{-} & =q^{4} X^{-} \tau
\end{aligned}
$$

and

$$
\begin{aligned}
\tau T^{3} & =T^{3} \tau \\
\tau T^{+} & =q^{-4} T^{+} \tau \\
\tau T^{-} & =q^{4} T^{-} \tau .
\end{aligned}
$$

The definition of the orbital angular momentum as it was given in Ref. [5] can be best formulated in terms of the elements

$$
\begin{aligned}
L^{+} & =\frac{1}{q^{2} \sqrt{1+q^{2}}} \tau^{-\frac{1}{2}} T^{+} \\
L^{-} & =-\frac{1}{q^{3} \sqrt{1+q^{2}}} \tau^{-\frac{1}{2}} T^{-} \\
L^{3} & =\frac{1}{q^{2}\left(1-q^{2}\right)}\left(\tau^{-\frac{1}{2}}-1-\frac{\lambda^{2}}{1+q^{2}} \vec{T}^{2}\right)
\end{aligned}
$$

As the $q$-generalization of the fact that orbital angular momentum is orthogonal to the coordinate vector we impose the constraint

$$
L \circ X=L^{3} X^{3}-q L^{+} X^{-}-\frac{1}{q} L^{-} X^{+}=0 .
$$

We shall see that this defines orbital angular momentum uniquely.

\section{The $t$ Algebra:}

The algebra introduced in the previous chapter allows a homomorphism of the $T$ algebra into the $X$ algebra. This was first seen in Ref. [6]. We find this homomorphism by interpreting Eqns. (2.5), (2.6) and (2.7) as inhomogeneous equations which can be solved for $T$ in terms of $X$. We first construct a particular solution $t$ and exhibit the homomorphism

$$
\begin{aligned}
& T^{+} \quad \mapsto \quad t^{+}=-\frac{1}{\lambda q^{3}} \sqrt{1+q^{2}} X^{+}\left(X^{3}\right)^{-1} \\
& T^{-} \quad \mapsto \quad t^{-}=\frac{q^{2}}{\lambda} \sqrt{1+q^{2}} X^{-}\left(X^{3}\right)^{-1} \\
& T^{3} \quad \mapsto \quad t^{3}=\frac{1}{\lambda}\left(1+R^{2}\left(X^{3}\right)^{-2}\right) .
\end{aligned}
$$

Here we extend the algebra by the inverse of $X^{3}$. To establish the homomorphism we have to use (2.1) to show that the $t$ elements satisfy (2.3). Furthermore the relations (2.5) to (2.7) are fullfilled by the $t$ elements. It is due to (2.2) that they satisfy (2.4) as well.

There are additional relations for the $t$ elements that follow from (2.1). They are

$$
\tau_{t}=1-\lambda t^{3}=-R^{2}\left(X^{3}\right)^{-2}
$$


and

$$
\begin{aligned}
& t^{+} t^{-}=-\frac{1}{\lambda^{2}}\left(1+q^{2} \tau_{t}\right) \\
& t^{-} t^{+}=-\frac{1}{\lambda^{2}}\left(1+\frac{1}{q^{2}} \tau_{t}\right) .
\end{aligned}
$$

It follows that the Casimir operator for the $t$ algebra takes a definite value and that in the notation of Appendix A, where $\bar{m}_{t}$ and $d_{t}$ are defined,

$$
\vec{T}^{2}=-\frac{1+q^{2}}{\lambda^{2}}, \quad \bar{m}_{t}=0, \quad d_{t}=-\frac{q^{2}}{\lambda} .
$$

This value of the Casimir operator and the sign of $\tau_{t}$, which is negative, show that the $t$ algebra cannot be represented by the well-known finite dimensional representations of the $T$ algebra [3]. In Appendix A we shall show that there are infinite-dimensional representations of the $T$ algebra among which there is one satisfying (3.2), (3.3) and (3.4). The representation is uniquely determined by these conditions, we present it here:

$$
\begin{aligned}
t^{3}\left|m_{t}\right\rangle= & \frac{1}{\lambda}\left(1+q^{2} q^{-4 m_{t}}\right)\left|m_{t}\right\rangle \\
t^{+}\left|m_{t}\right\rangle= & \frac{1}{\lambda q} \sqrt{q^{-4 m_{t}}-1}\left|m_{t}+1\right\rangle \\
t^{-}\left|m_{t}\right\rangle= & \frac{q}{\lambda} \sqrt{q^{-4\left(m_{t}-1\right)}-1}\left|m_{t}-1\right\rangle \\
& m_{t} \leq 0 .
\end{aligned}
$$

From $(3.5)$ it follows that

$$
t^{+}|0\rangle=0
$$

There is no state with positive $m_{t}$.

Eqns. (3.1) allow us to express the elements $X R^{-1}$ in terms of the $t$ elements:

$$
\begin{aligned}
X^{3} R^{-1} & = \pm\left(-\tau_{t}\right)^{-\frac{1}{2}} \\
X^{+} R^{-1} & =\mp \frac{\lambda q^{3}}{\sqrt{1+q^{2}}} t^{+}\left(-\tau_{t}\right)^{-\frac{1}{2}} \\
X^{-} R^{-1} & = \pm \frac{\lambda}{q^{2} \sqrt{1+q^{2}}} t^{-}\left(-\tau_{t}\right)^{-\frac{1}{2}}
\end{aligned}
$$

The two different signs are the signs of $X^{3} R^{-1}=\sqrt{\left(X^{3}\right)^{2} R^{-2}}$. These elements can be viewed as homogeneous coordinates in the $\mathbb{R}_{q}^{3}$ space.

The representations of these elements are now obtained from (3.5):

$$
\begin{aligned}
X^{3} R^{-1}\left|m_{t}\right\rangle & = \pm q^{2 m_{t}-1}\left|m_{t}\right\rangle \\
X^{+} R^{-1}\left|m_{t}\right\rangle & =\mp \frac{q}{\sqrt{1+q^{2}}} \sqrt{1-q^{4 m_{t}}}\left|m_{t}+1\right\rangle \\
X^{-} R^{-1}\left|m_{t}\right\rangle & = \pm \frac{1}{\sqrt{1+q^{2}}} \sqrt{1-q^{4\left(m_{t}-1\right)}}\left|m_{t}-1\right\rangle
\end{aligned}
$$

The different signs in (3.8) lead to inequivalent irreducible representations of the $X$ algebra. 


\section{The $K$ Algebra}

We continue to consider the Eqns. (2.5), (2.6) and (2.7) as inhomogeneous equations that should be solved for the T's. We have found one particular solution (3.1) and now move to the homogeneous part. This we do by the Ansatz:

$$
\begin{aligned}
T^{ \pm} & =\Delta^{ \pm}+t^{ \pm} \\
T^{3} & =\Delta^{3}+t^{3}
\end{aligned}
$$

Eqns (2.5), (2.6) and (2.7) become homogeneous equations for the $\Delta$ 's.

$$
\begin{aligned}
X^{ \pm} \Delta^{+} & =q^{ \pm 2} \Delta^{+} X^{ \pm} \\
X^{3} \Delta^{+} & =\Delta^{+} X^{3} \\
X^{ \pm} \Delta^{-} & =q^{ \pm 2} \Delta^{-} X^{ \pm} \\
X^{3} \Delta^{-} & =\Delta^{-} X^{3} \\
& \\
X^{ \pm} \Delta^{3} & =q^{ \pm 4} \Delta^{3} X^{ \pm} \\
X^{3} \Delta^{3} & =\Delta^{3} X^{3}
\end{aligned}
$$

These equations suggest the further Ansatz:

$$
\begin{aligned}
K^{ \pm} & = \pm\left(-\tau_{t}\right)^{-\frac{1}{2}} \Delta^{ \pm}, \\
K^{3} & =\left(\tau_{t}\right)^{-1} \Delta^{3} .
\end{aligned}
$$

The element $\tau_{t}$ satisfies the relation (2.11) and as a consequence all the $K$ s commute with all the $X \mathrm{~s}$ and therefore with all the $t$ 's as well

$$
\begin{aligned}
K^{A} X^{B} & =X^{B} K^{A} \\
K^{A} t^{B} & =t^{B} K^{A}
\end{aligned}
$$

Now we turn to (2.3) and compute the $K K$ relations:

$$
\begin{aligned}
q^{-1} K^{+} K^{-}-q K^{-} K^{+} & =K^{3} \\
q^{2} K^{3} K^{+}-q^{-2} K^{+} K^{3} & =\left(q+q^{-1}\right) K^{+} \\
-q^{-2} K^{3} K^{-}+q^{2} K^{-} K^{3} & =\left(q+q^{-1}\right) K^{-}
\end{aligned}
$$

This is exactly the same algebra as (2.3). Any realization of the $K$-algebra will lead to a realization of the $T$-algebra:

$$
\begin{aligned}
T^{ \pm} & =t^{ \pm} \pm\left(-\tau_{t}\right)^{\frac{1}{2}} K^{ \pm} \\
T^{3} & =t^{3}+\tau_{t} K^{3}
\end{aligned}
$$

This is a relation which is familiar from the comultiplication of two representations of the algebra (2.3):

$$
\begin{aligned}
\Delta_{\beta}\left(T^{3}\right) & =T^{3} \otimes 1+\tau \otimes T^{3} \\
\Delta_{\beta}\left(T^{ \pm}\right) & =T^{ \pm} \otimes 1 \pm \sqrt{-\tau} \otimes T^{ \pm}
\end{aligned}
$$

This comultiplication will be discussed in Appendix Q. It is adjusted to representations where the first factor has negative eigenvalues of $\tau$. We emphasize that the representations of the $t$ algebra in (4.8) are restricted by the relations (3.3) whereas for the $K$ algebra any representation would do as long as we are not considering any conjugation properties. 
If we now demand the conjugation property (2.4) for the $T$ algebra we find for the $K$ algebra:

$$
\overline{K^{3}}=K^{3}, \quad \overline{K^{+}}=-\frac{1}{q^{2}} K^{-} .
$$

Note the sign. The $K$ algebra belongs to the $S U_{q}(1,1)$ quantum group.

If we now use the condition (2.14) for orbital angular momentum we will specify the $K$ algebra representation uniquely as well. It needs some computation to express the $L$ algebra (2.13) in terms of the $t$ and $K$ algebras.

$$
\begin{aligned}
L^{+}= & \frac{1}{q^{2} \sqrt{1+q^{2}}}\left\{\left(-\tau_{t}\right)^{-\frac{1}{2}} t^{+} \otimes\left(-\tau_{k}\right)^{-\frac{1}{2}}+1 \otimes\left(-\tau_{k}\right)^{-\frac{1}{2}} K^{+}\right\} \\
L^{-}= & -\frac{1}{q^{3} \sqrt{1+q^{2}}}\left\{\left(-\tau_{t}\right)^{-\frac{1}{2}} t^{-} \otimes\left(-\tau_{k}\right)^{-\frac{1}{2}}-1 \otimes\left(-\tau_{k}\right)^{-\frac{1}{2}} K^{-}\right\} \\
L^{3}= & \frac{q^{2}-1}{q^{4}\left(q^{2}+1\right)}\left\{\frac{q^{2}}{\lambda^{2}}\left(-\tau_{t}\right)^{\frac{1}{2}} \otimes\left(-\tau_{k}\right)^{\frac{1}{2}}+\left(-\tau_{t}\right)^{\frac{1}{2}} \otimes\left(-\tau_{k}\right)^{-\frac{1}{2}}\left(-K^{+} K^{-}+\frac{q^{2}}{\lambda^{2}}\right)\right. \\
& \left.-\frac{1+q^{2}}{\lambda^{2}}\left(-\tau_{t}\right)^{-\frac{1}{2}} \otimes\left(-\tau_{k}\right)^{-\frac{1}{2}}+t^{-} \otimes\left(-\tau_{k}\right)^{-\frac{1}{2}} K^{+}-q^{2} t^{+} \otimes\left(-\tau_{k}\right)^{-\frac{1}{2}} K^{-}\right\}
\end{aligned}
$$

This already shows that we should restrict the representations such that $\tau_{k}$ has negative eigenvalues. There is an additional reason for it. We shall see in Appendix C that the coproduct (4.8) only leads to representations with positive eigenvalues of $\tau$ if $\tau_{k}$ has negative eigenvalues. Only in this case the representations of $T$ can be decomposed into finite-dimensional ones. We are here adding this as an additional assumption - not knowing if it is really necessary.

With this assumption it will follow from the comultiplication rule of Appendix $\mathrm{C}$ that we have to choose

$$
d_{k}=-\frac{1}{\lambda q^{2}} .
$$

Now we are ready to evaluate (2.14). This relation will be true if and only if:

$$
\underline{m}_{k}=-1 \text {. }
$$

This is in the notation of Appendix B. For the Casimir operator we find

$$
\vec{T}_{k}^{2}=-\frac{1+q^{2}}{\lambda^{2}}
$$

This uniquely determines the $K$ algebra representation. The generators of the orbital angular momentum will be denoted by $T_{\text {orb }}$.

We find the result:

$$
\begin{aligned}
& T_{\text {orb }}^{3}=t^{3} \otimes 1+\tau_{t} \otimes K^{3} \\
& T_{\text {orb }}^{ \pm}=t^{ \pm} \otimes 1 \pm \sqrt{-\tau_{t}} \otimes K^{ \pm}
\end{aligned}
$$

where the $t$ and $K$ representations are determined by (3.4) and (4.12), (4.13).

We can add spin to orbital angular momentum:

$$
\begin{aligned}
T^{3} & =T_{\text {orb }}^{3} \otimes 1+\tau_{\text {orb }} \otimes S^{3} \\
T^{ \pm} & =T_{\text {orb }}^{ \pm} \otimes 1+\sqrt{\tau_{\text {orb }}} \otimes S^{ \pm}
\end{aligned}
$$

The spin operators $S$ can be in any finite-dimensional representations of the $T$ algebra. 


\section{Representations of the $\boldsymbol{T}_{\text {orb }}$ Algebra:}

The representation of the $K$ algebra that enters orbital angular momentum is characterized by (4.12), (4.13) and (4.14):

$$
d_{k}=-\frac{1}{\lambda q^{2}}, \quad \underline{m}_{k}=-1, \quad \vec{T}_{k}^{2}=-\frac{1+q^{2}}{\lambda^{2}} .
$$

It is an infinite-dimensional representation with $m_{k}$ ranging from 0 to $\infty$.

$$
\begin{aligned}
K^{3}\left|m_{k}\right\rangle & =\frac{1}{\lambda}\left(1+\frac{1}{q^{2}} q^{-4 m_{k}}\right)\left|m_{k}\right\rangle \\
K^{+}\left|m_{k}\right\rangle & =\frac{1}{q \lambda} \sqrt{1-q^{-4\left(m_{k}+1\right)}}\left|m_{k}+1\right\rangle \\
K^{-}\left|m_{k}\right\rangle & =-\frac{q}{\lambda} \sqrt{1-q^{-4 m_{k}}}\left|m_{k}-1\right\rangle \\
K^{-}|0\rangle & =0, \quad m_{k} \geq 0
\end{aligned}
$$

The representation $T_{\text {orb }}$ of orbital angular momentum is the tensor product of this representation and the $t$ representation given in (3.5). The eigenstates of $T_{\text {orb }}^{3}$ are characterized by the two numbers $m_{t}$ and $m_{k}$.

$$
\begin{aligned}
T_{\text {orb }}^{3}\left|m_{t}, m_{k}\right\rangle= & \frac{1}{\lambda}\left(1-q^{-4\left(m_{t}+m_{k}\right)}\right)\left|m_{t}, m_{k}\right\rangle \\
T_{\text {orb }}^{+}\left|m_{t}, m_{k}\right\rangle= & \frac{1}{\lambda q} \sqrt{q^{-4 m_{t}}-1}\left|m_{t}+1, m_{k}\right\rangle \\
& +\frac{1}{\lambda} q^{-2 m_{t}} \sqrt{1-q^{-4\left(m_{k}+1\right)}}\left|m_{t}, m_{k}+1\right\rangle \\
T_{\text {orb }}^{-}\left|m_{t}, m_{k}\right\rangle= & \frac{q}{\lambda} \sqrt{q^{-4\left(m_{t}-1\right)}-1}\left|m_{t}-1, m_{k}\right\rangle \\
& +\frac{q^{2}}{\lambda} q^{-2 m_{t}} \sqrt{1-q^{-4 m_{k}}}\left|m_{t}, m_{k}-1\right\rangle
\end{aligned}
$$

In this representation $\vec{K}^{2}$ and $\vec{t}^{2}$ are diagonal. The transformation to the basis where $\vec{T}_{\text {orb }}^{2}$ is diagonal will be constructed in the next chapter. The value of $T_{\text {orb }}^{3}$ in (5.3) shows that we have found finite-dimensional representations of $T_{\text {orb }}$.

We obtain from (5.3)

$$
\tau_{\text {orb }}\left|m_{t}, m_{k}\right\rangle=q^{-4\left(m_{t}+m_{k}\right)}\left|m_{t}, m_{k}\right\rangle .
$$

The representation of the $X$ algebra can be obtained from (3.8). The element $R$ is central, it will be diagonal in the $m_{t}, m_{k}$ basis. We denote the eigenvalue of $R^{2}$ by

$$
R^{2}\left|m_{t}, m_{k}, M\right\rangle=q^{4 M+2} z_{0}^{2}\left|m_{t}, m_{k}, M\right\rangle,
$$

where $z_{0}$ is an arbitrary parameter characterizing the radius. Then we obtain from (3.8) the representation of $X^{3}$ :

$$
X^{3}\left|m_{t}, m_{k}, M\right\rangle=q^{2\left(m_{t}+M\right)} z_{0}\left|m_{t}, m_{k}, M\right\rangle .
$$

We have absorbed the sign in (3.8) yielding inequivalent representations in the sign of $z_{0}$ which is not determined by (5.5).

This and (5.4) suggest that we should introduce a notation characterizing the eigenvalue of $X^{3}$ by a quantum number as well as the eigenvalue of $T_{\text {orb }}^{3}$.

$$
\nu=m_{t}+M, \quad m=m_{t}+m_{k}
$$


In this notation we obtain the representation which was also found in [7]:

$$
\begin{aligned}
X^{3}|M, \nu, m\rangle= & q^{2 \nu} z_{0}|M, \nu, m\rangle \\
R^{2}|M, \nu, m\rangle= & q^{4 M+2} z_{0}^{2}|M, \nu, m\rangle \\
T_{\text {orb }}^{3}|M, \nu, m\rangle= & \frac{1}{\lambda}\left(1-q^{-4 m}\right)|M, \nu, m\rangle \\
X^{+}|M, \nu, m\rangle= & -\frac{q^{2} z_{0}}{\sqrt{1+q^{2}}} \sqrt{q^{4 M}-q^{4 \nu}}|M, \nu+1, m+1\rangle \\
X^{-}|M, \nu, m\rangle= & \frac{q z_{0}}{\sqrt{1+q^{2}}} \sqrt{q^{4 M}-q^{4(\nu-1)}}|M, \nu-1, m-1\rangle \\
T_{\text {orb }}^{+}|M, \nu, m\rangle= & \frac{1}{q^{2}-1} \sqrt{q^{4(M-\nu)}-1}|M, \nu+1, m+1\rangle \\
& +\frac{1}{\lambda} \sqrt{q^{4(M-\nu)}-q^{-4(m+1)}}|M, \nu, m+1\rangle \\
T_{\text {orb }}^{-}|M, \nu, m\rangle= & \frac{q^{2}}{q^{2}-1} \sqrt{q^{4(M-\nu+1)}-1}|M, \nu-1, m-1\rangle \\
& +\frac{q^{2}}{\lambda} \sqrt{q^{4(M-\nu)}-q^{-4 m}}|M, \nu, m-1\rangle \\
& \nu \leq M, m \geq \nu-M
\end{aligned}
$$

\section{Reduction of the representation of $T_{\text {orb }}$}

The above representation (5.3) of $T_{\text {orb }}$ is a tensor product of two representations, with $\vec{t}^{2}$ and $\vec{K}^{2}$ diagonal. We proceed with its decomposition into a sum of irreducible representations characterized by the eigenvalues of $\vec{T}_{\text {orb }}^{2}$. From the Appendix $A$ we know that for $d=\lambda d_{t} d_{k}=\lambda^{-1}$ the eigenvalues of $\vec{T}^{2}$ are $q[l][l+1]$. Therefore we start with an Ansatz of the form

$$
\begin{aligned}
|l, m\rangle & =\sum_{m_{k}, m_{t}} c_{l, m}^{m_{k}, m_{t}}\left|m_{t}, m_{k}\right\rangle, \quad m_{k} \geq 0, m_{t} \leq 0 \\
\vec{T}_{\text {orb }}^{2}|l, m\rangle & =q[l][l+1]|l, m\rangle .
\end{aligned}
$$

According to (5.7) $m=m_{t}+m_{k}$, so that we have

$$
c_{l, m}^{m_{k}, m_{t}}=c_{l, m}^{m_{t}} \delta_{m, m_{t}+m_{k}} .
$$

From the definition (2.9) of $\vec{T}^{2}$ and the Equations (5.3) we obtain a recursion relation for the coefficients $c_{l, m}^{m_{t}}$.

$$
\begin{aligned}
& \left(q^{2 l+2}+q^{-2 l}-\left(q^{2}+1\right) q^{2(m+1)-4 m_{t}}\right) c_{l, m}^{m_{t}}= \\
& q^{2 m+1}\left(\sqrt{\left(q^{-4 m_{t}}-1\right)\left(q^{-4 m_{t}}-q^{-4 m}\right)} c_{l, m}^{m_{t}+1}\right. \\
& \left.\quad+\sqrt{\left(q^{4-4 m_{t}}-1\right)\left(q^{4-4 m_{t}}-q^{-4 m}\right)} c_{l, m}^{m_{t}-1}\right)
\end{aligned}
$$

A comparison with the $q$-difference Equation (D.18) for the functions $\widetilde{P}_{m}^{l}$ defined in (D.8) and (D.16) shows that (b.3) is solved by

$$
c_{l, m}^{m_{t}}= \begin{cases}q^{m_{t}-m-1} \sqrt{1-q^{-2}} \widetilde{P}_{l}^{m}\left( \pm q^{2\left(m_{t}-1\right)-2 m}\right) & \text { for } m \geq 0 \\ q^{m_{t}-1} \sqrt{1-q^{-2}} \widetilde{P}_{l}^{|m|}\left( \pm q^{2\left(m_{t}-1\right)}\right) & \text { for } m<0\end{cases}
$$

Note that $P_{l}^{m}$ is defined for $m \geq 0$ only. 
The orthogonality condition (D.19) for the functions $\widetilde{P}_{l}^{m}$ suggests to start with the direct sum of two representations of the form (5.3), such that both signs of the argument of $\widetilde{P}_{l}^{m}$ appear.

$$
\begin{aligned}
|l, m\rangle & =\sum_{\sigma= \pm 1} \sum_{m_{t}} c_{l, m}^{m_{t}, \sigma}\left|m_{t}, m_{k}, \sigma\right\rangle \\
c_{l, m}^{m_{t}, \sigma} & = \begin{cases}\sqrt{1-q^{-2}} q^{m_{t}-1-m} \widetilde{P}_{l}^{m}\left(\sigma q^{2\left(m_{t}-m-1\right.}\right) & \text { for } m \geq 0 \\
\sqrt{1-q^{-2}} q^{m_{t}-1} \widetilde{P}_{l}^{|m|}\left(\sigma q^{2\left(m_{t}-1\right)}\right) & \text { for } m<0\end{cases}
\end{aligned}
$$

We know that $m_{k} \geq 0, m_{t} \leq 0$ and $m=m_{t}+m_{k}$, thus $m_{t}$ is restricted by $m_{t} \leq 0$ and $m \geq m_{t}$. The last condition comes into effect for negative values of $m$. Note that if $m_{t}$ takes its largest allowed value, the coefficient of $c_{l, m}^{m_{t}+1}$ in (6.3) vanishes. We are free to choose this $c$ to be zero. For $m \geq 0$ it then follows from (6.3) that $c_{l, m}^{m_{t}}=0$ for $m_{t}>0$ and for $m<0$ the same is true for $m_{t}>m$.

The values of $l$ are restricted by the condition $|m| \leq l$, as seen from (D.9). This is obviously consistent with the recursion formula (6.3).

We have chosen the normalization in (6.6) in such a way that according to (D.19) the eigenfunctions of $\vec{T}_{\text {orb }}^{2}$ are orthonormal:

$$
\begin{aligned}
& q^{-1} \lambda \sum_{\sigma= \pm 1} \sum_{m_{t}=-\infty}^{\min \{0, m\}} q^{2\left(m_{t}-1\right)-m-|m|} \widetilde{P}_{l}^{|m|}\left(\sigma q^{2\left(m_{t}-1\right)-m-|m|}\right) \widetilde{P}_{l^{\prime}}^{|m|}\left(\sigma q^{2\left(m_{t}-1\right)-m-|m|}\right) \\
& =\delta_{l, l^{\prime}}
\end{aligned}
$$

To see this for $m<0$ it is enough to shift the summation variable $m_{t} \rightarrow m_{t}+m$.

We now assume that the two representations with $\sigma=+1$ and $\sigma=-1$ also lead to a different sign of $z_{0}$ in (5.6).

$$
X^{3}\left|m_{t}, m_{k}, M, \sigma\right\rangle=q^{2\left(m_{t}+M\right)} \sigma\left|z_{0}\right|\left|m_{t}, m_{k}, M, \sigma\right\rangle
$$

Then it follows from (E.7) that the functions $\widetilde{P}_{l}^{m}$ satisfy the following completeness relation

$$
\begin{aligned}
& q^{-1} \lambda \sum_{l=0}^{\infty} q^{m_{t}+m_{t}^{\prime}-2-m-|m|} \widetilde{P}_{l}^{|m|}\left(\sigma q^{2\left(m_{t}-1\right)-m-|m|}\right) \widetilde{P}_{l}^{|m|}\left(\sigma^{\prime} q^{2\left(m_{t}^{\prime}-1\right)-m-|m|}\right) \\
& =\delta_{\sigma, \sigma^{\prime}} \delta_{m_{t}, m_{t}^{\prime}}
\end{aligned}
$$

This construction shows that for fixed $m, \vec{T}_{o r b}^{2}$ is a selfadjoint operator in the basis $\left|m_{t}, m_{k}, \sigma\right\rangle, \sigma= \pm 1$, and that the transformation from the basis $\left|m_{t}, m_{k}, \sigma\right\rangle$ to the basis $|l, m\rangle$ is an isometry.

\section{Appendices}

\section{A Representations of the $T$ Algebra}

When constructing representations of the $T$ algebra, we are aiming at representations where $T^{3}$ is selfadjoint (or essentially selfadjoint). This allows us to assume $T^{3}$ to be diagonal:

$$
T^{3}|m\rangle=f(m)|m\rangle .
$$

The eigenvalue of $T^{3}$ is $f(m), m$ is a labelling of the eigenstates. 
The second equation of (2.3) shows that $T^{+}|m\rangle$ is again an eigenstate of $T^{3}$, we choose the labelling such that this state is labelled by $m+1$ :

$$
T^{+}|m\rangle=c_{m}|m+1\rangle .
$$

The relation (2.3) leads to a recursion formula for $f(m)$ :

$$
f(m+1)=\frac{1}{q^{4}} f(m)+\frac{1}{q^{2}}\left(q+q^{-1}\right) .
$$

This recursion formula has the solution

$$
f(m)=\frac{1}{\lambda}-d q^{-4 m}
$$

From $\overline{T^{3}}=T^{3}$ follows that $d q^{-4 m}$ has to be real. We take $d$ and $m$ to be real.

For the operator $\tau$ of (2.10) follows

$$
\tau|m\rangle=\lambda d q^{-4 m}|m\rangle .
$$

From the conjugation properties of $T^{+}$it follows that

$$
T^{-}|m\rangle=q^{2} c_{m-1}^{*}|m-1\rangle .
$$

The third equation of (2.3) is the conjugate of the second one. The first equation of (2.3) amounts to a recursion formula for $c_{m}^{*} c_{m}$

$$
q c_{m-1}^{*} c_{m-1}-q^{3} c_{m}^{*} c_{m}=f(m) .
$$

This recursion formula can be solved:

$$
c_{m}^{*} c_{m}=\frac{1}{\lambda}\left\{-\frac{1}{q^{2} \lambda}+\alpha \lambda q^{-2 m}-\frac{d}{q^{4}} q^{-4 m}\right\} .
$$

The real parameter $\alpha$ is not determined by (A.7).

We see that $c_{m}^{*} c_{m}$ becomes negative for $m \rightarrow \infty$. This is not allowed. There has to be a largest value of $m$, say $\bar{m}$, such that

$$
c_{\bar{m}}^{*} c_{\bar{m}}=0 .
$$

Then it follows from (A.2) that $T^{+}$does not lead to a state with a larger value than $\bar{m}$. To analyze this situation we introduce the function:

$$
\begin{aligned}
x & =q^{-2 m} \\
h(x) & =\left\{-\frac{1}{q\left(q^{2}-1\right)}+\alpha \lambda x-\frac{d}{q^{4}} x^{2}\right\}
\end{aligned}
$$

The function $h(x)$ is negative for $x=0$, the sign of $h(x)$ for $x \rightarrow \infty$ depends on the sign of $d$. In any case $h(x)$ has to have a zero for positive $x$ to represent $c^{*} c$. We have to demand

$$
x_{1}=q^{-2 \bar{m}}, \quad h\left(x_{1}\right)=0 .
$$

The parameter $\alpha$ can now be expressed in terms of $\bar{m}$.

$$
\alpha=\frac{1}{\lambda}\left\{\frac{1}{\lambda} q^{2(\bar{m}-1)}+d q^{-2(\bar{m}+2)}\right\} .
$$

If $\alpha$ takes this value $h(x)$ has the two zeros:

$$
x_{1}=q^{-2 \bar{m}}, \quad x_{2}=\frac{1}{\lambda d} q^{2(\bar{m}+1)} .
$$


We obtain for $c^{*} c$ :

$$
c_{m}^{*} c_{m}=-\frac{d}{\lambda q^{4}}\left(q^{-2 m}-q^{-2 \bar{m}}\right)\left(q^{-2 m}-\frac{1}{\lambda d} q^{2(\bar{m}+1)}\right) .
$$

The representation is characterized by two parameters, $\bar{m}$ and $d$. We use this parameter in the explicit form of the matrix elements:

$$
\begin{aligned}
T^{3}|m\rangle & =\left(\frac{1}{\lambda}-d q^{-4 m}\right)|m\rangle \\
T^{+}|m\rangle & =\frac{1}{q^{2}} \sqrt{\frac{d}{\lambda}\left(q^{-2 m}-q^{-2 \bar{m}}\right)\left(\frac{1}{\lambda d} q^{2(\bar{m}+1)}-q^{-2 m}\right)}|m+1\rangle \\
T^{-}|m\rangle & =\sqrt{\frac{d}{\lambda}\left(q^{-2(m-1)}-q^{-2 \bar{m}}\right)\left(\frac{1}{\lambda d} q^{2(\bar{m}+1)}-q^{-2(m-1)}\right)}|m-1\rangle \\
\tau|m\rangle & =d \lambda q^{-4 m}|m\rangle \\
\vec{T}^{2} & =\frac{1}{\lambda^{2} \sqrt{\lambda d}}\left(q^{2(\bar{m}+1)}+\lambda d q^{-2 \bar{m}}\right)-\frac{1+q^{2}}{\lambda^{2}}
\end{aligned}
$$

Let us now have a closer look at the condition $c^{*} c \geq 0$. For this purpose we discuss the three cases $d>0, d=0$ and $d<0$ separately.

$d>0$

There has to be a smallest value of $\mathrm{m}$, say $\underline{m}$, such that $|\underline{m}\rangle \neq 0$ and $T^{-}|\underline{m}\rangle=0$, therefore

$$
c_{\underline{m}-1}^{*} c_{\underline{m}-1}=0 .
$$

From (A.14) follows for $\bar{m} \geq 0$

$$
d=\frac{1}{\lambda}, \quad \underline{m}=-\bar{m} .
$$

The number of states between $\bar{m}$ and $\underline{m}$ has to be integer:

$$
2 \bar{m}+1 \equiv n \text {. }
$$

This shows that $\bar{m}$ has to be integer or half integer and we found the $2 l+1$ dimensional representation $(\bar{m}=l)$ of $s o_{q}(3)$.

$d=0$

In this case $h(x)$ is a linear function:

$$
h(x)=-\frac{1}{q\left(q^{2}-1\right)}+\alpha \lambda x .
$$

Now $\alpha$ has to be positive for $h$ to have a zero for positive $x$. From A.12 follows

$$
\alpha=\frac{1}{q^{2} \lambda^{2}} q^{2 \bar{m}}
$$

The representation can be obtained from (A.15). The parameter $\bar{m}$ that characterizes the representation can take any real value. The representation is infinitedimensional, however, $\tau$ is not invertible.

$d<0$

This is the situation that arises for the $t$ algebra, as can be seen from (3.2). In this case $x_{2}$ is negative. We only have a largest value of $m$. The representation 
is infinite-dimensional and $\bar{m}$ is not restricted. The matrix elements are obtained from (A.15). We write them such as to exhibit the positive square roots:

$$
\begin{aligned}
T^{3}|m\rangle & =\left(\frac{1}{\lambda}-d q^{-4 m}\right)|m\rangle \\
T^{+}|m\rangle & \left.=\frac{1}{q^{2}} \sqrt{-\frac{d}{\lambda}} \sqrt{\left(q^{-2 m}-q^{-2 \bar{m}}\right)\left(q^{-2 m}-\frac{1}{\lambda d} q^{2(\bar{m}+1)}\right)}|m+1\rangle \quad \text { A. } 21\right) \\
T^{-}|m\rangle & =\sqrt{-\frac{d}{\lambda}} \sqrt{\left(q^{-2(m-1)}-q^{-2 \bar{m}}\right)\left(q^{-2(m-1)}-\frac{1}{\lambda d} q^{2(\bar{m}+1)}\right)}|m-1\rangle \\
\tau|m\rangle & =d \lambda q^{-4 m}|m\rangle
\end{aligned}
$$

$\tau$ has negative eigenvalues only, thus $\tau^{\frac{1}{2}}$ and $\vec{T}^{2}$ will not be real.

\section{B Representations of the $K$ Algebra}

The algebraic relations of the $K$ algebra are the same as the relations of the $T$ algebra, they are different only as a $*$ algebra:

$$
\begin{aligned}
\overline{K^{3}} & =K^{3}, & \overline{K^{+}} & =-\frac{1}{q^{2}} K^{-} \\
\overline{T^{3}} & =T^{3}, & \overline{T^{+}} & =\frac{1}{q^{2}} T^{-}
\end{aligned}
$$

This makes the $K$ algebra a $s u_{q}(1,1)$ algebra.

All the results that depend only on the algebraic relations are the same as for the $T$ algebra.

$$
\begin{array}{llll}
\text { (A.1) } & K^{3}|m\rangle & =\phi(m)|m\rangle \\
\text { (A.2) }: & K^{+}|m\rangle & =\gamma_{m}|m\rangle \\
\text { (A.4) }: & \phi(m) & =\frac{1}{\lambda}-d_{k} q^{-4 m}
\end{array}
$$

We again take $d_{k}$ and $m$ real.

$$
\text { (A.5) }: \tau_{k}|m\rangle=\lambda d_{k} q^{-4 m}|m\rangle
$$

For $K^{-}$there is a change in sign due to (B.1):

$$
\begin{array}{ll}
\text { (A.6) : } & K^{-}|m\rangle=-q^{2} \gamma_{m-1}^{*}|m-1\rangle \\
\text { A.8) : } & \gamma_{m}^{*} \gamma_{m}=-\frac{1}{\lambda}\left\{-\frac{1}{q^{2} \lambda}+\alpha \lambda q^{-2 m}-\frac{d_{k}}{q^{4}} q^{-4 m}\right\}
\end{array}
$$

Now $\gamma_{m}^{*} \gamma_{m}$ becomes positive for $m \rightarrow \infty$, we do not have to cut off the spectrum at a largest value of $m$. We shall see that all the representations are infinite-dimensional.

We introduce the function $\kappa(x)$ analogous to $h(x)$ in (A.10):

$$
\kappa(x)=\left\{\frac{1}{q^{2} \lambda^{2}}-\alpha x+\frac{d_{k}}{\lambda q^{4}} x^{2}\right\}, \quad x=q^{-2 m} .
$$

The representations of the $K$ algebra are:

$$
\begin{aligned}
K^{3}|m\rangle & =\left(\frac{1}{\lambda}-d_{k} q^{-4 m}\right)|m\rangle \\
K^{+}|m\rangle & =\sqrt{\kappa\left(q^{-2 m}\right)}|m+1\rangle \\
K^{-}|m\rangle & =-q^{2} \sqrt{\kappa\left(q^{-2(m-1)}\right)}|m-1\rangle
\end{aligned}
$$


They are characterized by $\alpha$ and $d_{k}$ and restricted by the condition $\kappa\left(q^{-2 m}\right) \geq 0$. To discuss this condition we determine the zeros of $\kappa(x)$

$$
\begin{aligned}
\kappa\left(x_{1,2}\right) & =0 \\
x_{1,2} & =\frac{\lambda}{2 d_{k} q^{-4}}\left\{\alpha \pm \sqrt{\alpha^{2}-4 d_{k} q^{-6} \lambda^{-3}}\right\}
\end{aligned}
$$

We discuss the cases $d_{k}>0, d_{k}=0$ and $d_{k}<0$ separately and start with $d_{k}>0$ :

In this case $\kappa(x)$ has no positive zero for $\alpha<2 q^{-3} \sqrt{d_{k} \lambda^{-3}}=\alpha_{0}$. The range of $m$ is not restricted, it can be of the form $m_{0}+n, n \in \mathbb{Z}_{0}$. If $\alpha \geq \alpha_{0}$ we will have two positive zeros and $\kappa(x)$ can be written in the form

$$
\kappa(x)=\frac{1}{q^{2} \lambda^{2} x_{1} x_{2}}\left(x-q^{-2 \bar{m}}\right)\left(x-q^{-2 \underline{m}}\right) .
$$

The values of the zeros $x_{1}, x_{2}$ determine the parameters $\alpha$ and $d_{k}$ and therefore the representation.

$$
\begin{aligned}
d_{k} & =\frac{q^{2}}{\lambda} q^{2(\bar{m}+\underline{m})} \\
\alpha & =\frac{1}{q^{2} \lambda^{2}}\left(q^{2 \underline{m}}+q^{2 \bar{m}}\right)
\end{aligned}
$$

There are now two inequivalent representations. We find that in one representation the allowed values of $m$ are

$$
m \leq \bar{m}, \quad m=\bar{m}, \bar{m}-1, \bar{m}-2, \ldots
$$

For the other representation we find

$$
m>\underline{m}, \quad m=\underline{m}+1, \underline{m}+2, \ldots
$$

Now we consider

$d_{k}=0$ :

The function $\kappa$ becomes linear. It is positive at $x=0$ and, depending on $\alpha$, stays positive or becomes negative for $x \rightarrow \infty$. If $\alpha<0$ there is no restriction in the range of $m, m=m_{0}+n, n \in \mathbb{Z}$. If $\alpha>0$ there is a lowest eigenvalue of $m$, we are at the situation of (B.14).

Finally we consider

$d_{k}<0$ :

In this case $\kappa(x)$ is positive for $x \rightarrow 0$ and negative for $x \rightarrow \infty$. There is one zero for $x>0$. This can also be seen from (B.10) because the square root will now be larger than $\alpha$. The relevant zero of $\kappa(x)$ is:

$$
\begin{aligned}
x_{1} & =\frac{\lambda}{2\left|d_{k}\right| q^{-4}}\left\{-\alpha+\sqrt{\alpha^{2}+4\left|d_{k}\right| q^{-6} \lambda^{-3}}\right\} \\
& =q^{-2 \underline{m}_{k}}
\end{aligned}
$$

Now all values of $\alpha$ are allowed. The range of $m$ will be as in (B.14).

For orbital angular momentum we encounter the representation

$$
d_{k}=-\frac{1}{q^{2} \lambda}, \quad x_{1}=q^{2}
$$

This leads to $\alpha=0$ and

$$
\kappa\left(q^{-2 m}\right)=\frac{1}{\lambda^{2} q^{2}}\left(1-q^{-4(m+1)}\right) .
$$

The respective representation is shown in (5.2). 


\section{Comultiplication}

The standard comultiplication rule for the algebra (2.3) is:

$$
\begin{aligned}
\Delta\left(T^{3}\right) & =T^{3} \otimes 1+\tau \otimes T^{3} \\
\Delta\left(T^{ \pm}\right) & =T^{ \pm} \otimes 1+\tau^{\frac{1}{2}} \otimes T^{ \pm}
\end{aligned}
$$

As a consequence, $\tau$ is group-like:

$$
\Delta(\tau)=\tau \otimes \tau
$$

The algebra (2.3) is the same for the $T$ algebra and the $K$ algebra, they are distinguished by their conjugation properties (B.1).

As long as $\tau^{\frac{1}{2}}$ is hermitean, (C.1) will respect the conjugation properties and we have a comultiplication within the $T$ algebra or the $K$ algebra respectively. From (C.2) follows that $\Delta\left(\tau^{\frac{1}{2}}\right)$ will be hermitean if $\tau^{\frac{1}{2}}$ is.

$$
\Delta\left(\tau^{\frac{1}{2}}\right)=\tau^{\frac{1}{2}} \otimes \tau^{\frac{1}{2}}
$$

If $\tau^{\frac{1}{2}}$ is not hermitean $\Delta(T)$ will have no definite conjugation properties even if $T$ has.

We now turn to the product of representations as it follows from the comultiplication rule (C.1). If we have two representations of the algebra (2.3) we obtain a new one by the rule

$$
\begin{aligned}
\Delta\left(T^{3}\right) & =T_{1}^{3} \otimes 1+\tau_{1} \otimes T_{2}^{3} \\
\Delta\left(T^{ \pm}\right) & =T_{1}^{ \pm} \otimes 1+\tau_{1}^{\frac{1}{2}} \otimes T_{2}^{ \pm}
\end{aligned}
$$

From the discussion above follows that we can multiply two representations of the $T$ algebra ( $K$ algebra) to obtain a representation of the $T$ algebra ( $K$ algebra) as

long as $\tau_{1}^{\frac{1}{2}}$ is hermitean. From now on we shall drop the indices 1 and 2 again, first and second representations will be defined by the position in the product (C.4).

That the $\tau^{\frac{1}{2}}$ of the first representation is hermitean means $d_{1}>0$. We shall discuss this situation first.

$d_{1}>0$ :

The product of two representations of the $T$ algebra ( $K$ algebra) will be a $T$ algebra ( $K$ algebra). From (C.2 follows

$$
d=\lambda d_{1} d_{2} .
$$

If $d_{2}$ is negative $d$ will be negative as well.

For the $T$ algebra $d$ positive restricts $d$ to be $d=\frac{1}{\lambda}$. This characterizes the finite-dimensional representations. From (C.5) follows that the product of two finite-dimensional representations is finite-dimensional as expected but also that the product of a finite-dimensional representation $\left(d_{1}=\frac{1}{\lambda}\right)$ with an infinite-dimensional representation $\left(d_{2}<0\right)$ leads to $d<0$ and cannot be reduced to finite-dimensional representations.

For the $K$ algebra all representations are infinite-dimensional.

We now turn to the case that $d_{1}$ is negative, $\tau_{1}^{\frac{1}{2}}$ will be anti-hermitean. $d_{1}<0$ :

In this case the product of two representations will in general not have welldefined conjugation properties. We can, however, start from a modified comultiplication rule:

$$
\begin{aligned}
\Delta_{\beta}\left(T^{3}\right) & =T^{3} \otimes 1+\tau \otimes T^{3} \\
\Delta_{\beta}\left(T^{ \pm}\right) & =T^{ \pm} \otimes 1 \pm(-\tau)^{\frac{1}{2}} \otimes T^{ \pm}
\end{aligned}
$$


If $(-\tau)^{\frac{1}{2}}$ is hermitean this rule allows us to multiply a representation of the $T(K)$ algebra by a representation of the $K(T)$ algebra to obtain a $T(K)$ algebra.

$$
\begin{aligned}
& T^{3} \otimes 1+\tau \otimes K^{3} \\
& T^{ \pm} \otimes 1 \pm(-\tau)^{\frac{1}{2}} \otimes K^{ \pm}
\end{aligned}
$$

will be a representation of the $T$ algebra whereas

$$
\begin{aligned}
& K^{3} \otimes 1+\tau_{k} \otimes T^{3} \\
& K^{ \pm} \otimes 1 \pm\left(-\tau_{k}\right)^{\frac{1}{2}} \otimes T^{ \pm}
\end{aligned}
$$

will be a representation of the $K$ algebra.

For the comultiplication $(\overline{C .6}) \tau$ will be group-like as well and it follows again that

$$
d=\lambda d_{1} d_{2}
$$

But now $d_{1}$ is negative.

Of special interest is the case that $d_{1}$ and $d_{2}$ are both negative, then $d$ is positive. If we multiply $T \times K$ to obtain a $T$ algebra then we know that $d=\frac{1}{\lambda}$ and, as a consequence

$$
d_{1} d_{2}=\frac{1}{\lambda^{2}}
$$

to obtain a representation with well-defined conjugation properties. This is exactly the case for the construction of the $T_{\text {orb }}$ algebra in the main part of this paper.

\section{The big $q$-Jacobi polynomials}

In this appendix we recall some basics about $q$-special functions [8], [9], [10], in particular the big $q$-Jacobi polynomials.

First, we introduce some useful notation. The expressions

$$
[a]=\frac{q^{a}-q^{-a}}{q-q^{-1}} \stackrel{q \rightarrow 1}{\rightarrow} a, \quad[a] !=\prod_{k=1}^{a}[k] \stackrel{q \rightarrow 1}{\rightarrow} a !
$$

are known as symmetric $q$-numbers and symmetric $q$-factorials respectively. The corresponding $q$-binomial coefficient is

$$
\left[\begin{array}{l}
n \\
k
\end{array}\right]= \begin{cases}\frac{[n] !}{[k] ![n-k] !} & \text { for } n \geq k, \\
0 & \text { for } n<k \text { or } n, k<0 .\end{cases}
$$

Of course $\left[\begin{array}{l}n \\ k\end{array}\right] \stackrel{q \rightarrow 1}{\rightarrow}\left(\begin{array}{l}n \\ k\end{array}\right)$. There are also "unsymmetric" counterparts of these objects: the basic $q$-number

$$
\frac{1-q^{a}}{1-q} \stackrel{q \rightarrow 1}{\rightarrow} a
$$

and the $q$-shifted factorial (Pochammer-symbol)

$$
(a ; q)_{k}=\prod_{n=0}^{k-1}\left(1-a q^{n}\right), \quad\left(a_{1}, a_{2}, \ldots, a_{i} ; q\right)_{k}=\prod_{m=1}^{i}\left(a_{m} ; q\right)_{k} .
$$

The Jackson integral of a function $f(x)$ is defined for $q>1$ by

$$
\int_{0}^{a} d_{q^{-1}} x f(x)=\left(1-q^{-1}\right) \sum_{\nu=0}^{\infty} a q^{-\nu} f\left(a q^{-\nu}\right)
$$


With the help of the $q$-shifted factorials, the basic hypergeometric function can be introduced

$$
\begin{aligned}
{ }_{r} \phi_{s} & \left(\begin{array}{c}
a_{1}, \ldots, a_{r} \\
b_{1}, \ldots, b_{s}
\end{array} \mid q^{-1} ; x\right)= \\
& \sum_{k=0}^{\infty} \frac{\left(a_{1}, \ldots, a_{r} ; q^{-1}\right)_{k}}{\left(b_{1}, \ldots, b_{s} ; q^{-1}\right)_{k}}(-1)^{(1+s-r) k} q^{-\frac{1}{2}(1+s-r) k(k-1)} \frac{x^{k}}{\left(q^{-1} ; q^{-1}\right)_{k}}
\end{aligned}
$$

This series plays in the theory of $q$-special functions a role analogous to that of the hypergeometric series in the theory of usual special functions. We have considered a base $q^{-1}$ here, because in this way ${ }_{r} \phi_{s}$ is well-defined for $q>1$, which is the case we are interested in here.

The big $q$-Jacobi polynomials [8], [9] are constructed in terms of the basic hypergeometric series as

$$
P_{l}\left(x ; a, b, c ; q^{-1}\right)={ }_{3} \phi_{2}\left(\begin{array}{l}
q^{l}, a b q^{-(l+1)}, x \\
a q^{-1}, c q^{-1}
\end{array} \mid q^{-1} ; q^{-1}\right) .
$$

For the applications we consider in this paper, we are interested in the case

$$
\begin{aligned}
P_{l}^{m}(x) & \equiv P_{l-m}\left(x ; q^{-2 m}, q^{-2 m},-q^{-2 m} ; q^{-2}\right), \quad m \geq 0 \\
= & \sum_{k=0}^{l-m}(-1)^{k} \frac{q^{-k(m+1)}\left(x ; q^{-2}\right)_{k}}{\left(-q^{-2(m+1)} ; q^{-2}\right)_{k}}\left[\begin{array}{l}
l-m \\
k
\end{array}\right]\left[\begin{array}{l}
l+m+k \\
k
\end{array}\right]\left[\begin{array}{l}
m+k \\
k
\end{array}\right]^{-1}
\end{aligned}
$$

Notice that the $P_{l}^{m}$ are polynomials of the order $l-m$ in $x$. Due to the factor $\left[\begin{array}{l}l-m \\ k\end{array}\right]$, which vanishes for $k>l-m$ according to the definition (D.2). The sum in (D.6) actually becomes finite. Due to the same factor the polynomials $P_{l}^{m}$ vanish, if the condition $m \leq l$ is not satisfied:

$$
P_{l}^{m}(x)=0 \text { for } l<m .
$$

The further condition $m \geq 0$ is necessary for the polynomials $P_{m}^{l}$ to be well-defined, due to the factor $\left(q^{-2(m-1)} ; q^{-2}\right)_{k}$ in the denominator of the basic hypergeometric function which otherwise vanishes for negative $m$.

Some of the first big $q$-Jacobi polynomials are

$$
\begin{aligned}
P_{0}^{0}(x) & =1, & P_{1}^{0}(x) & =x, \\
P_{2}^{0}(x) & =\frac{1}{q[2]}\left([3] x^{2}-q^{-2}\right), & P_{3}^{0}(x) & =\frac{x}{q^{5}[2]}\left([5] q^{2} x^{2}-[3]\right), \\
P_{1}^{1}(x) & =1, & P_{2}^{1}(x) & =x, \\
P_{3}^{1}(x) & =\frac{1}{q^{5}[4]}\left(q^{4}[5] x^{2}-1\right) . & &
\end{aligned}
$$

From Ref. 8] and [9] we learn that the polynomials $P_{l}^{m}(x)$ satisfy a recurrence relation

$$
x q^{m}[2 l+1] P_{l}^{m}(x)=q^{l}[l+m+1] P_{l+1}^{m}(x)+q^{-l-1}[l-m] P_{l-1}^{m}(x)
$$

a $q$-difference equation

$$
\begin{gathered}
\left(q^{-1-2 m}\left(q^{2 l+1}+q^{-2 l-1}\right) x^{2}-q^{-4(m+1)}\left(q^{2}+1\right)\right) P_{l}^{m}(x)= \\
q^{-2(2 m+1)}\left(x^{2}-1\right) P_{l}^{m}\left(x q^{-2}\right)+\left(x^{2}-q^{-4(m+1)}\right) P_{l}^{m}\left(x q^{2}\right)
\end{gathered}
$$


and the orthonormality condition

$$
\int_{-q^{-2(m+1)}}^{q^{-2(m+1)}} d_{q^{-2}} x w_{l}^{m}(x) w_{l^{\prime}}^{m}(x) P_{l}^{m}(x) P_{l^{\prime}}^{m}(x)=\delta_{l, l^{\prime}} .
$$

Here, the weight function $w_{l}^{m}$ is defined by

$$
\begin{aligned}
w_{l}^{m}(x) \equiv & \sqrt{\frac{\left(q^{-4(m+1)} ; q^{-4}\right)_{\infty}}{\left(q^{-4}, q^{-4(m+1)-2} ; q^{-4}\right)_{\infty}\left(-q^{-2} ; q^{-2}\right)_{\infty}}} \sqrt{\frac{[2 m+1]}{2[2 l+1]}} \\
& \times q^{-\frac{1}{2}\left(l^{2}+l+2 l m-3 m^{2}+m+3\right)} \sqrt{\left(x^{2} q^{4 m} ; q^{-4}\right)_{m}} \sqrt{\frac{\left(q^{-2} ; q^{-2}\right)_{l-m}}{\left(q^{-2(2 m+1)} ; q^{-2}\right)_{l-m}}}
\end{aligned}
$$

Actually, as it is done e.g. in [9], the big $q$-Jacobi polynomials can be alternatively defined as those polynomials in $x$ which are orthonormal with respect to the Jackson integral with the weight function $w_{l}^{m}(x)$ in (D.14).

The weight function has the following scaling properties

$$
\begin{aligned}
w_{l}^{m}\left(x q^{-2}\right) & =w_{l}^{m}(x) \sqrt{\frac{\left(1-x^{2}\right)}{\left(1-x^{2} q^{4 m}\right)}}, \\
w_{l-1}^{m}(x) & =w_{l}^{m}(x) q^{l} \sqrt{\frac{[l+m][2 l+1]}{[l-m][2 l-1]}} .
\end{aligned}
$$

It is useful for the purposes of this paper to absorb the weight function in the definition of the polynomials themselves and to introduce the further notation

$$
\widetilde{P}_{l}^{m}(x)=w_{l}^{m}(x) P_{l}^{m}(x) .
$$

With the help of (D.15) it turns out that (D.11) and (D.12) become respectively

$$
x q^{m+1} \widetilde{P}_{l}^{m}(x)=\sqrt{\frac{[l-m+1][l+m+1]}{[2 l+1][2 l+3]}} \widetilde{P}_{l+1}^{m}(x)+\sqrt{\frac{[l+m][l-m]}{[2 l+1][2 l-1]}} \widetilde{P}_{l-1}^{m}(x)
$$

and

$$
\begin{gathered}
\left(\left(q^{2 l+1}+q^{-2 l-1}\right) q^{-1} x^{2}-\left(q^{2}+1\right) q^{-2(m+2)}\right) \widetilde{P}_{l}^{m}(x)= \\
q^{-2(m+1)} \sqrt{\left(x^{2}-1\right)\left(x^{2} q^{4 m}-1\right)} \widetilde{P}_{l}^{m}\left(x q^{-2}\right) \\
+\sqrt{\left(x^{2}-q^{-4(m+1)+1}\right)\left(x^{2}-q^{-4}\right)} \widetilde{P}_{l}^{m}\left(x q^{2}\right)
\end{gathered}
$$

By using (D.13) and the definition of the Jackson integral (D.5) we obtain the following orthonormality condition for the functions $\widetilde{P}_{l}^{m}(x)$

$$
\left(1-q^{-2}\right) \sum_{\sigma= \pm 1} \sum_{n=-\infty}^{0} q^{2(n-m-1)} \widetilde{P}_{l}^{m}\left(\sigma q^{2(n-m-1)}\right) \widetilde{P}_{l^{\prime}}^{m}\left(\sigma q^{2(n-m-1)}\right)=\delta_{l, l^{\prime}}
$$

Moreover, the functions $\widetilde{P}_{l}^{m}(x)$ have the property that they transform under a parity transformation like

$$
\widetilde{P}_{l}^{m}(-x)=(-1)^{l-m} \widetilde{P}_{l}^{m}(x) .
$$

In the particular case $m=0$ the big $q$-Jacobi polynomials become the big $q$-Legendre polynomials, which in the limit $q \rightarrow 1$ yield the usual Legendre polynomials. In the same limit from the polynomials $P_{l}^{m}(x)$ we recover the Jacobi polynomials with the normalization $P_{l}^{m}(1)=1$. 


\section{E Diagonalization of $X^{3}$}

In this appendix we study the transformation which is inverse to the transformation (6.5), (6.6) constructed in Section 5. We show how the big $q$-Jacobi polynomials can be used to diagonalize $X^{3}$ in the basis where $\vec{T}_{\text {orb }}^{2}, T_{\text {orb }}^{3}, R^{2}$ are diagonal. 13.

The representation where $\vec{T}_{\text {orb }}^{2}, T_{\text {orb }}^{3}, R^{2}$ are diagonal can be found in [11, [12],

$$
\begin{aligned}
\vec{T}_{\text {orb }}^{2}|M, l, m\rangle=q[l][l+1] & |M, l, m\rangle \\
X^{3}|M, l, m\rangle=r_{0} q^{2 M+m} & \left\{\sqrt{\frac{[l+m+1][l-m+1]}{[2 l+1][2 l+3]}}|M, l+1, m\rangle\right. \\
& \left.+\sqrt{\frac{[l+m][l-m]}{[2 l+1][2 l-1]}}|M, l-1, m\rangle\right\} \\
X^{+}|M, l, m\rangle=r_{0} q^{2 M+m} & \left\{q^{-l} \sqrt{\frac{[l+m+1][l+m+2]}{[2][2 l+1][2 l+3]}}|M, l+1, m+1\rangle\right. \\
& \left.-q^{l+1} \sqrt{\frac{[l-m][l-m-1]}{[2][2 l+1][2 l-1]}}|M, l-1, m+1\rangle\right\} \\
X^{-}|M, l, m\rangle=r_{0} q^{2 M+m} & \left\{q^{l} \sqrt{\frac{[l-m+1][l-m+2]}{[2][2 l+1][2 l+3]}}|M, l+1, m-1\rangle\right. \\
& \left.-q^{-l-1} \sqrt{\frac{[l+m][l+m-1]}{[2][2 l+1][2 l-1]}}|M, l-1, m-1\rangle\right\}
\end{aligned}
$$

where

$$
0 \leq l<\infty, \quad-l \leq m \leq l
$$

We make the following Ansatz for an eigenfunction of $X^{3}$

$$
X^{3} \sum_{M, l, m} d_{M, l, m}|M, l, m\rangle=z \sum_{M, l, m} d_{M, l, m}|M, l, m\rangle,
$$

with $z$ the corresponding eigenvalue. By using (E.2) we obtain a recursion relation for the coefficients $d_{M, l, m}$

$$
\begin{aligned}
z d_{M, l, m}=r_{0} \frac{q^{2 M+m}}{\sqrt{[2 l+1]}}\{ & \sqrt{\frac{[l-m+1][l+m+1]}{[2 l+3]}} d_{M, l+1, m} \\
& \left.+\sqrt{\frac{[l+m][l-m]}{[2 l-1]}} d_{M, l-1, m}\right\} .
\end{aligned}
$$

A comparison with the recursion relation $(\widetilde{D .17})$ for the functions $\widetilde{P}_{l}^{m}$ defined in (D.16) in terms of the Jacobi polynomials shows that a solution of (E.4) is

$$
d_{M, l, m}^{\nu, \sigma}= \begin{cases}\sqrt{1-q^{-2}} q^{\nu-M-1-m} \widetilde{P}_{l}^{m}\left(\sigma q^{2(\nu-M-1-m)}\right) & \text { for } m \geq 0 \\ \sqrt{1-q^{-2}} q^{\nu-M-1} \widetilde{P}_{l}^{|m|}\left(\sigma q^{2(\nu-M-1)}\right) & \text { for } m<0\end{cases}
$$

where $z=\sigma r_{0} q^{-1+2 \nu}, \sigma= \pm 1$. By comparing with the form of the eigenvalues of $X^{3}$ (5.8) we see that we have to restrict

$$
\nu, M \in \mathbb{Z}, \quad \nu \leq M, \quad m \geq \nu-M .
$$


Notice that the argument of the functions would correspond to $x=\cos \theta=\frac{z}{r}$ classically, apart from the $q$-factor $q^{-(m+|m|)} \rightarrow 1$ for $q \rightarrow 1$.

$X^{3}$ is a self-adjoint operator in this representation. This was shown in Ref. [14].

Now, the set of eigenfunctions of a self-adjoint operator is complete, therefore we expect a completeness relation to hold for the eigenfunctions of $X^{3}$. In fact, (D.19) can be interpreted in this way. As the sum (D.19) contains two sums, one where the argument of $P_{m}^{l}$ is positive and one where it is negative, we obtain a representation where the eigenvalues of $X^{3}$ can have both signs, so that we automatically find the direct sum of two representations of the type (5.8). The normalization of the coefficients $d_{M, l, m}$ in (E.5) has been chosen in such a way as to yield exactly (D.19).

As the eigenfunctions of a selfadjoint operator corresponding to different eigenvalues are orthogonal, since the normalization constant is already fixed by (D.19), we argue that the following relation holds

$$
\left(1-q^{-2}\right) \sum_{l=0}^{\infty} q^{\nu+\nu^{\prime}-2} \widetilde{P}_{l}^{|m|}\left(\sigma q^{2(\nu-1)}\right) \widetilde{P}_{l}^{|m|}\left(\sigma^{\prime} q^{2\left(\nu^{\prime}-1\right)}\right)=\delta_{\nu, \nu^{\prime}} \delta_{\sigma, \sigma^{\prime}}
$$

where $\sigma, \sigma^{\prime}= \pm 1$ are the signs of the argument of the functions and $\nu \leq \min \{m, 0\}$. This is an interesting result for itself about the Jacobi polynomials.

\section{References}

[1] A. Dimakis and J. Madore, Differential calculi and linear connections, J. Math. Phys. 37, 4647 (1996);

M. Dubois-Violette, R. Kerner, and J. Madore, Gauge bosons in a noncommutative geometry, Phys. Lett. B217, 485 (1989);

B. Jurčo, P. Schupp, Noncommutative Yang-Mills from equivalence of star products, hep-th/0001032.

J. Madore, S. Schraml, P. Schupp, J. Wess, Gauge Theory on Noncommutative Spaces, hep-th/0001203;

N. Seiberg, E. Witten, String Theory and Noncommutative Geometry, JHEP 9909, 032, (1999), hep-th/9908142.

[2] L. Faddeev, N. Reshetikhin, and L. Takhtajan, Quantization of Lie groups and Lie algebras, Leningrad Math. J. 1193 (1990)

[3] J. Wess, q-deformed Heisenberg Algebras, lectures given at the 8.Internationale Universitätswochen für Kern- und Teilchenphysik, Schladming, Austria, to appear in the proceedings, preprint math-ph/9910013, Oct. 1999

[4] H. Snyder, Quantized space-time, Phys. Rev. 7138 (1947).

[5] A. Lorek, W. Weich, J. Wess, Non-commutative Euclidean and Minkowski Structures, Z.Phys. C 76: 375-386, (1997)

[6] B.L. Cerchiai, G. Fiore, J. Madore, Geometrical Tools for Quantum Euclidean Spaces, preprint LMU-TPW 99-17, MPI-PhT/99-45, Dip. Mat. Appl. Napoli 99-52, (1999)

[7] G. Fiore, The Euclidean Hopf Algebra $U_{q}\left(E^{N}\right)$ and its fundamental Hilbert Space Representations, J. Math. Phys. 36:4363-4405, (1995)

[8] R. Koekoek, R. F. Swarttouw, The Askey-Scheme of hypergeometric orthogonal polynomials and its q-analogue, Report-no OP-SF 20 Feb 1996, preprint math.CA/9602214, (1996) 
[9] T.H. Koornwinder, Compact Quantum Groups and q-special functions, in 'Representations of Lie Groups and Quantum Groups', V. Baldoni, M. A. Picardello (Eds.), Pitman Research Notes in Math. Series 311: 46-128, Longman scientific \& Technical, 1994

[10] G. Gasper, M. Rahman, Basic Hypergeometric Series, Encyclopedia of Mathematics and Its Applications 35, Cambridge University Press, Cambridge, 1990

[11] B. L. Cerchiai, J. Wess, q-deformed Minkowski Space based on a $q$-Lorentz Algebra, E. Phys. J. C 5: 553-566, (1998)

[12] B.-D. Dörfel, Non-commutative Euclidean structures in compact spaces, preprint hep-th/9907136, (1999)

[13] S. Schraml, Dreidimensionale q-deformierte Quantenmechanik, Diplomarbeit, Universität München, (1998)

[14] W. Weich, The Hilbert Space Representations for $\mathrm{SO}_{q}(3)$ symmetric Quantum Mechanics, preprint LMU-TPW 94-5, hep-th/9404029, (1994) 